\title{
On Improving the Effectiveness of Adversarial Training
}

\author{
Yi Qin \\ Colorado School of Mines \\ Golden, Colorado \\ yiqin@mines.edu
}

\author{
Ryan Hunt \\ Colorado School of Mines \\ Golden, Colorado \\ ryhunt@mines.edu
}

\author{
Chuan Yue \\ Colorado School of Mines \\ Golden, Colorado \\ chuanyue@mines.edu
}

\begin{abstract}
Machine learning models, including neural networks, are vulnerable to adversarial examples, which are adversarial inputs generated from legitimate examples by applying small perturbations to fool machine learning models to misclassify. Algorithms that are used to generate adversarial examples are called adversarial example generation methods. As the state-of-the-art defense approach, adversarial training improves the robustness of machine learning models by augmenting the training data with adversarial examples. However, adversarial training is far from being perfect yet, and a deeper understanding of it is always needed for further improving its effectiveness. In this paper, we propose to investigate two research questions. The first question is: whether Method-Based Ensemble Adversarial Training (MBEAT) could be beneficial, i.e., whether leveraging the adversarial examples generated by multiple methods could help increase the effectiveness of adversarial training. The second question is: whether Round Gap Of Adversarial Training (RGOAT) could exist, i.e., whether a neural network model adversarially trained in one round would not be robust against the adversarial examples further generated from this model itself. We design an adversarial training experimental framework to answer these two research questions. We find that MBEAT is indeed beneficial, indicating that it has some important value in practice. We also find that RGOAT indeed exists, indicating that adversarial training should be an iterative process.
\end{abstract}

\section{KEYWORDS}

Adversarial Examples, Adversarial Training, Adversarial Machine Learning, Neural Networks, Deep Learning

ACM Reference Format:

Yi Qin, Ryan Hunt, and Chuan Yue. 2019. On Improving the Effectiveness of Adversarial Training. In Fifth ACM International Workshop on Security and Privacy Analytics (IWSPA '19), March 27, 2019, Richardson, TX, USA. ACM, New York, NY, USA, 10 pages. https://doi.org/10.1145/3309182.3309190

\section{INTRODUCTION}

Deep neural networks are able to directly learn from raw data and can outperform traditional machine learning models. As a result, they have been increasingly used in a variety of application domains such as image classification, natural language processing,

Permission to make digital or hard copies of all or part of this work for personal or classroom use is granted without fee provided that copies are not made or distributed for profit or commercial advantage and that copies bear this notice and the full citation on the first page. Copyrights for components of this work owned by others than ACM must be honored. Abstracting with credit is permitted. To copy otherwise, or republish, to post on servers or to redistribute to lists, requires prior specific permission and/or a fee. Request permissions from permissions@acm.org.

IWSPA '19, March 27, 2019, Richardson, TX, USA

(c) 2019 Association for Computing Machinery.

ACM ISBN 978-1-4503-6178-1/19/03 ..\$15.00

https://doi.org/10.1145/3309182.3309190 and malware detection. However, machine learning models especially deep neural networks are demonstrated to be vulnerable to adversarial examples at the test time $[2,16,19]$.

Adversarial examples are malicious inputs generated to fool machine learning models to misclassify such as misclassifying images of pandas as gibbons or images of stop signs as yield signs, but they are still often correctly identified by humans as the images representing the original objects such as pandas or stop signs. This could be a serious potential threat in many important systems such as the object and traffic sign recognition systems of self-driving cars and robots. In deep learning, with the knowledge of a neural network architecture and its parameters, adversarial examples can be generated by many attack methods such as Fast Gradient Sign Method (FGSM) [6], Jacobian-based Saliency Map Approach (JSMA) [16], Basic Iterative Method (BIM) [8], and L-BFGS [19].

The state-of-the-art defense approach is adversarial training [6, 19]. Its basic idea is to increase the robustness of a machine learning model by adding adversarial examples to the training set. Although this approach is reasonable and intuitive, it still has many limitations such as not being effective against multi-step attacks [9]. A deeper understanding of this approach is always needed for further improving its effectiveness.

In this paper, we propose to investigate two research questions. The first question is: whether Method-Based Ensemble Adversarial Training (MBEAT) could be beneficial, i.e., whether leveraging the adversarial examples generated by multiple methods could help increase the effectiveness of adversarial training. Note that traditional ensemble adversarial training [20] focuses more on the ensemble of adversarial examples from different neural network models, while MBEAT is the ensemble of adversarial examples generated by different attack methods. The second question is: whether Round Gap Of Adversarial Training (RGOAT) could exist, i.e., whether a neural network model adversarially trained in one round would not be robust against the adversarial examples further generated from this model itself. By investigating these two questions, we aim to achieve a deeper understanding of adversarial training thus potentially helping researchers further improve its effectiveness or find better defense solutions against adversarial examples.

We design an adversarial training experimental framework to answer these two research questions. In more details, we first train a clean model as a baseline. Next, we use four representative attack methods (FGSM, JSMA, BIM, and L-BFGS) to generate adversarial examples from the clean model, and train 15 MBEAT models with the different combinations of the four attack methods. These two steps will help us answer the first research question about MBEAT. After that, adversarial training is performed for the second round and the third round to help us answer the second research question about RGOAT. Our experimental results show that MBEAT is indeed beneficial, indicating that it has some important value in practice. 
Our experimental results also show that RGOAT indeed exists, indicating that adversarial training should be an iterative process.

The main contributions of this paper are: (1) we propose to investigate two research questions regarding adversarial training; (2) we design an adversarial training experimental framework to answer the two research questions; (3) we show that MBEAT is beneficial and RGOAT exists to help researchers further improve the effectiveness of adversarial training.

The rest of the paper is structured as follows: Section 2 introduces the related work on adversarial example generation and adversarial training, Section 3 describes our adversarial training experimental framework, Section 4 presents our experiments and results, Section 5 discusses the limitations of this work and potential future work, and Section 6 concludes the paper.

\section{RELATED WORK}

Szegedy et al. [19] first defined adversarial examples as malicious inputs generated from legitimate examples by applying small perturbations to fool machine learning models to misclassify. In deep learning, with the knowledge of a neural network architecture and its parameters, adversarial examples can be generated by many powerful attack methods.

Goodfellow et al. [6] proposed Fast Gradient Sign Method (FGSM) which aims to generate adversarial examples fast without constraining too much about the number and size of perturbations on an input image. Papernot et al. [16] designed Jacobian-based Saliency Map Approach (JSMA) to reduce the number and size of perturbations at the cost of more intensive computation. Kurakin et al. [8] applied FGSM multiple times at a finer granularity and referred to this method as Basic Iterative Method (BIM). Szegedy et al. [19] proposed the L-BFGS method to find the solution of a gradient-based optimization problem. Besides these four representative methods, many other attack methods have been proposed by researchers [1, 3-5, 12, 17, 21].

Szegedy et al. [19] proposed a state-of-the-art defense approach called adversarial training, which improves the robustness of machine learning models by augmenting the training data with adversarial examples. However, adversarial training is far from being perfect yet. For example, Kurakin et al. [9] showed that adversarial training is not effective against multi-step attacks. Because the adversarial example generation process is often based on certain machine learning model and adversarial examples may transfer between models, Tramer et al. [20] introduced the model-based ensemble adversarial training technique to increase the effectiveness of adversarial training. This technique basically augments the training data with the adversarial examples generated from multiple pre-trained neural network models. This technique is useful because it takes the network model factor into the consideration of the ensemble; however, it does not consider the generation method or algorithm factor in the ensemble.

Overall, adversarial training is still the state-of-the-art defense approach, it has limitations, and there are opportunities to improve it. Due to these reasons, in this work we aim to explore more undiscovered properties of adversarial learning, and achieve a deeper understanding of it thus potentially helping researchers further improve its effectiveness or find better defense solutions against adversarial examples.

\section{ADVERSARIAL TRAINING EXPERIMENTAL FRAMEWORK}

Recall that the goal of this work is to answer the following two research questions. Question 1: whether Method-Based Ensemble Adversarial Training (MBEAT) could be beneficial, i.e., whether leveraging the adversarial examples generated by multiple methods could help increase the effectiveness of adversarial training. Question 2: whether Round Gap Of Adversarial Training (RGOAT) could exist, i.e., whether a neural network model adversarially trained in one round would not be robust against the adversarial examples further generated from this model itself.

We now introduce the threat model that we consider and the adversarial training experimental framework that we design for answering these two research questions. The following terms are used throughout this paper: source examples are the legitimate inputs used by the attack methods to generate the adversarial examples, the legitimate class of a source example such as the stop sign is referred to as the source class, and an adversarial example aims to fool machine learning models to misclassify it as a different target class such as the yield sign.

\subsection{Threat Model}

We consider a typical computer vision classification task, in which a neural network model $h$ is trained to map or label an input image $x$ to a certain class $y$. Quite often, the model $h$ produces a class score vector with the length as the total number of possible classes, and $y$ is the class with the highest score.

Adversarial Goal. For a given model $h$ and a source example with the known class represented as $(x, y)$, an adversary's goal is to perform a targeted attack [16], which is to generate an adversarial example $x_{a d v}$ with certain perturbation constraint $\epsilon$ such that $x_{a d v}$ is close to $x$ and is still perceived by humans as $y$ but will be misclassified by $h$ as the target class $y_{t} \neq y$. Targeted attacks are most desirable for attackers because they may incur the severest consequences such as misclassifying stop signs as yield signs. Formally, given a source example $(x, y)$ and the perturbation constraint $\epsilon$, an adversary aims to generate an adversarial example $x_{a d v}$ as shown in Formula 1:

$$
\arg \min _{x-x_{a d v}}\left\|x-x_{a d v}\right\|_{p}
$$

such that $h\left(x_{a d v}\right)=y_{t}$ and $\left\|x-x_{a d v}\right\|_{p} \leq \epsilon$. Here $\|\cdot\|_{p}$ represents the $l_{p}$ norm $(p \in[0, \infty))$ as defined in Formula 2 which is for measuring the distance between $x$ and $x_{a d v}$.

$$
\|v\|_{p}=\left(\sum_{i=1}^{n}\left|v_{i}\right|^{p}\right)^{1 / p}
$$

Four commonly used norms for adversarial example generation are: $l_{0} l_{1}, l_{2}$, and $l_{\infty}$. In more details, $l_{0}$ measures the number of different features (i.e., pixels in an image) between $x$ and $x_{a d v}, l_{1}$ measures the standard Manhattan distance between $x$ and $x_{a d v}$, $l_{2}$ measures the standard Euclidean distance between $x$ and $x_{a d v}$, and $l_{\infty}$ measures the maximum change to any of the features. 
Adversarial Capability. For the targeted attacks, we further consider that an adversary has the white-box attack capability. That is, the adversary has the knowledge of the neural network model and its parameters used by a defender. Specifically, the adversary knows the structure of the neural network such as its layers and activation functions. The adversary does not have the access to the training data but is able to obtain some source examples with true labels. These source examples are either from the legitimate test set or from the real world. The adversary will then generate adversarial examples based on the known network model and source examples by using certain attack methods or algorithms.

This white-box attack capability is a reasonable assumption in many scenarios especially because neural network models are sometimes not considered as confidential and indeed many well pre-trained models are widely shared over the Internet (e.g., by researchers and large companies) for being reused by anyone. Alternatively, Papernot et al. $[14,15]$ demonstrated the feasibility of a black-box attack where an adversary does not know the details about the neural network model. The authors trained a surrogate model with the query instances as training data and generated adversarial examples from the surrogate model. Due to the transferability property of adversarial examples, black-box attacks can still be successful in many cases. In this work, we focus on investigating the two research questions in the white-box attack setting, and we leave the investigation of the two questions in the black-box attack setting as a future work.

\subsection{Experimental Framework}

In order to answer the two research questions, we design an adversarial training experimental framework as shown in Figure 1. There are four steps in this framework: clean training, Method-Based Ensemble Adversarial Training (MBEAT), second adversarial training, and third adversarial training. First, we train a clean model as a baseline. Next, we use four representative attack methods (FGSM, JSMA, BIM, and L-BFGS) to generate adversarial examples from the clean model, and train 15 MBEAT models with the different combinations of the four attack methods. These two steps will help us answer the first research question about MBEAT. After that, adversarial training is performed for the second round and the third round to help us answer the second research question about RGOAT. More details are as follows.

Step 1: Clean Training. The goal of this step is to train a clean model as a baseline for generating adversarial examples from it. The workflow of clean training is shown in Figure 1a.

The training set is denoted as clean_train, and it will be used to train a clean model denoted as Model A. Model A is tested on a test set denoted as clean_test to evaluate its accuracy. Meanwhile, four representative adversarial example generation methods (FGSM, JSMA, BIM, and L-BFGS) are used on Model A to generate two different adversarial example sets $a d v \_A \_S 1$ and $a d v \_A \_S 2$ based on the same source examples. Later, adv_A_S1 will be used for training, while adv_A_S2 will be used for testing.

Step 2: MBEAT. This step is designed to answer the first research question about MBEAT. Besides, this step also helps us answer the second research question about RGOAT together with Step 3 and Step 4.
As shown in Figure 1b, we use the clean training set clean_train and the adversarial set adv_A_S1 to train Model B. Since we choose four methods, 15 different Models B are trained with MBEAT. Table 1 lists the notations of these 15 MBEAT models. For example, Model B_M8 means the training data used are clean_train and the adversarial examples generated from JSMA and BIM in adv_A_S1. Then these MBEAT models are tested on the clean test set clean_test and the adversarial set adv_A_S2. The four adversarial example generation methods are further used on these MBEAT models to generate two new different adversarial example sets $a d v \_B \_S 1$ and $a d v \_B \_S 2$ based on the same source examples.

Step 3: Second Adversarial Training. This is the second round adversarial training. It uses the clean training set clean_train, the adversarial set adv_A_S1 generated from the clean Model A, and the adversarial set adv_B_S1 generated from some first round adversarially trained Model B to train Model C as shown in Figure 1c. Note that we do not consider the method-based ensemble in this second round adversarial training. Instead, we only consider adversarial examples generated from the same method because the purpose of this step is to answer the second research question about RGOAT without being interfered with by MBEAT.

In other words, four different Models $\mathrm{C}$ are trained with the second adversarial training. Table 2 lists the notations of these four adversarially trained models in the second round. For example, model C_M2 means the training data used are clean_train and the adversarial examples generated from JSMA in both adv_A_S1 and adv_B_S1. Then these four models are tested on the clean test set clean_test and the adversarial sets adv_A_S2 and adv_B_S2. The four adversarial example generation methods are further used on these four models to generate two new different adversarial example sets $a d v_{-} C_{-} S 1$ and $a d v_{-} C_{-} S 2$ based on the same source examples.

Step 4: Third Adversarial Training. This is the third round adversarial training. The goal of this step is to further answer the second research question about RGOAT. As shown in Figure 1d, we use the clean training set clean_train and the adversarial sets adv_A_S1, adv_B_S1, and adv_C_S1 to train Model D. Four different Models D are trained with the third adversarial training. Still, we only consider the same method combination across sets adv_A_S2, adv_B_S2, and adv_C_S2. Table 3 lists the notations of these four adversarially trained models in the third round. Then these four models are tested on the clean test set clean_test and the adversarial sets adv_A_S2, adv_B_S2, and adv_C_S2. The four adversarial example generation methods are further used on these four models to generate one new adversarial example set $a d v \_D \_S 1$.

While we can continue this process to have more rounds of adversarial training, it is not necessary because the round 2 and round 3 results are sufficient for us to show the existence of RGOAT and raise the attention of researchers to this limitation in adversarial training.

\section{EXPERIMENTS AND RESULTS}

In this section, we first describe the experimental setup. Then, we present and analyze the experimental results to answer the two research questions. 


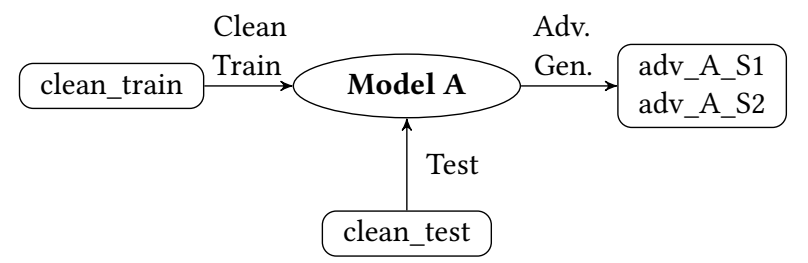

(a) Step 1: Clean Training. We use a clean training set clean_train to train a clean Model A. Then Model A is tested on a clean test set clean_test. Adversarial example generation methods are used on Model A to generate two different adversarial example sets adv_A_S1 and adv_A_S2.

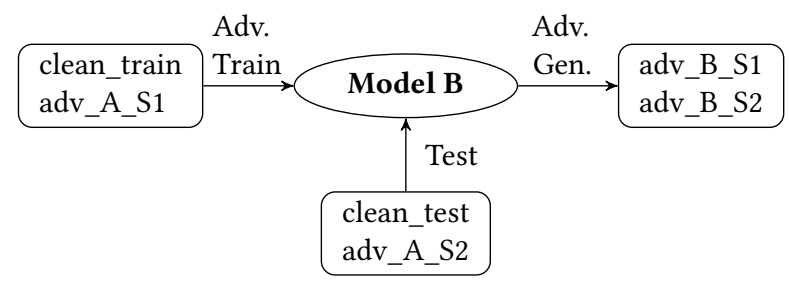

(b) Step 2: MBEAT. We use the clean training set clean_train and the adversarial set adv_A_S1 to train Model B. Since we choose four methods, 15 different Models B are trained with MBEAT. Then these 15 models are tested on the clean test set clean_test and the adversarial set adv_A_S2. Adversarial example generation methods are used on Model B to further generate two new different adversarial example sets adv_B_S1 and adv_B_S2.

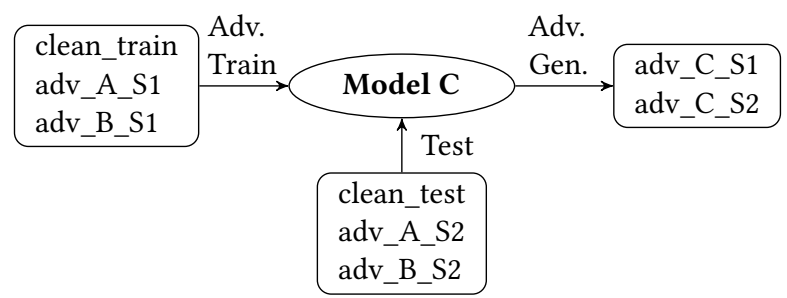

(c) Step 3: Second Adversarial Training. We use the clean training set clean_train and adversarial sets adv_A_S1 and adv_B_S1 to train Model C. Four different Models $\mathrm{C}$ are trained with the second adversarial training. Then these four models are tested on the clean test set clean test and adversarial sets adv_A_S2 and adv_B_S2. Adversarial example generation methods are applied on Model $\mathrm{C}$ to further generate two new different adversarial example sets adv_C_S1 and adv_C_S2.

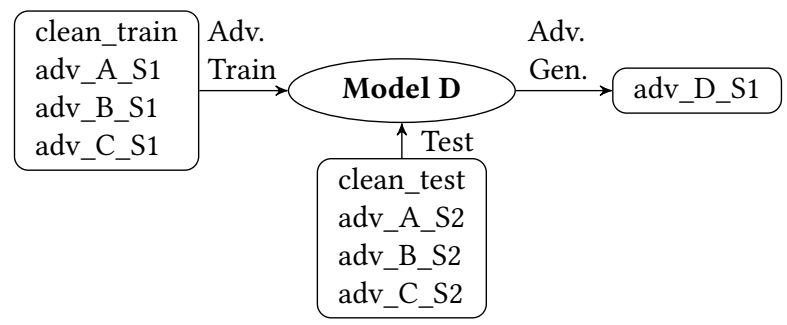

(d) Step 4: Third Adversarial Training. We use the clean training set clean_train and adversarial sets adv_A_S1, adv_B_S1, and adv_C_S1 to train Model D. Four different Models D are trained with the third adversarial training. Then these four models are tested on the clean test set clean_test and adversarial sets adv_A_S2, adv_B_S2, and adv_C_S2. Adversarial example generation methods are applied on Model $D$ to further generate a new adversarial example set adv_D_S1.

Figure 1: Adversarial Training Experimental Framework.
Table 1: Notations for the 15 MBEAT Models B. means that the adversarial examples generated by the corresponding attack method are included in the ensemble training, while $\mathrm{o}$ means they are not included.

\begin{tabular}{|c|cccc|}
\hline \multirow{2}{*}{ Notation } & \multicolumn{4}{|c|}{ Methods Used in the Ensemble } \\
\cline { 2 - 5 } & FGSM & JSMA & BIM & L-BFGS \\
\hline B_M1 & $\bullet$ & 0 & 0 & 0 \\
B_M2 & 0 & $\bullet$ & 0 & 0 \\
B_M3 & 0 & 0 & $\bullet$ & 0 \\
B_M4 & 0 & 0 & 0 & $\bullet$ \\
B_M5 & $\bullet$ & $\bullet$ & 0 & 0 \\
B_M6 & $\bullet$ & 0 & $\bullet$ & 0 \\
B_M7 & $\bullet$ & 0 & 0 & $\bullet$ \\
B_M8 & 0 & $\bullet$ & $\bullet$ & 0 \\
B_M9 & 0 & $\bullet$ & 0 & $\bullet$ \\
B_M10 & 0 & 0 & $\bullet$ & $\bullet$ \\
B_M11 & $\bullet$ & $\bullet$ & $\bullet$ & 0 \\
B_M12 & $\bullet$ & $\bullet$ & 0 & $\bullet$ \\
B_M13 & $\bullet$ & 0 & $\bullet$ & $\bullet$ \\
B_M14 & 0 & $\bullet$ & $\bullet$ & $\bullet$ \\
B_M15 & $\bullet$ & $\bullet$ & $\bullet$ & $\bullet$ \\
\hline
\end{tabular}

Table 2: Notations for the four Models C.

\begin{tabular}{|c|c|c|}
\hline \multirow{2}{*}{ Notation } & \multicolumn{2}{|c|}{ Adversarial Examples Used } \\
\cline { 2 - 3 } & adv_A_S1 & adv_B_S1 \\
\hline adv_C_M1 & FGSM & FGSM \\
adv_C_M2 & JSMA & JSMA \\
adv_C_M3 & BIM & BIM \\
adv_C_M4 & L-BFGS & L-BFGS \\
\hline
\end{tabular}

Table 3: Notations for the four Models D.

\begin{tabular}{|c|c|c|c|}
\hline \multirow{2}{*}{ Notation } & \multicolumn{3}{|c|}{ Adversarial Examples Used } \\
\cline { 2 - 4 } & adv_A_S1 & adv_B_S1 & adv_C_S1 \\
\hline adv_D_M1 & FGSM & FGSM & FGSM \\
adv_D_M2 & JSMA & JSMA & JSMA \\
adv_D_M3 & BIM & BIM & BIM \\
adv_D_M4 & L-BFGS & L-BFGS & L-BFGS \\
\hline
\end{tabular}

\subsection{Experimental Setup}

4.1.1 Dataset. We use the MNIST handwritten digits dataset [11] in this work. This dataset is considered as one of the most popular image datasets for researchers to benchmark their work. It consists of images for digits from 0 to 9 with a training set of 60,000 examples, and a test set of 10,000 examples. Each of the examples is a grayscale image with $28 \times 28$ pixels. Adversarial examples are generated from the source examples in the test set.

4.1.2 Neural Network Model. We choose the well-known LeNet5 [10] as the neural network model in this work. More advanced network models such as AlexNet [7] and GoogLeNet [18] for large image datasets such as ImageNet leverage many techniques of 
Table 4: Model Architecture for LeNet-5. We use learning rate $=0.001$, batch size $=128$, and number of epochs $=6$ in the training. The accuracy for the trained clean Model A is $99.05 \%$.

\begin{tabular}{cccc}
\hline Layer Type & $\begin{array}{c}\text { Input } \\
\text { Size }\end{array}$ & $\begin{array}{c}\text { Layer } \\
\text { Parameters }\end{array}$ & $\begin{array}{c}\text { Output } \\
\text { Size }\end{array}$ \\
\hline Input Layer & $28 \times 28 \times 1$ & - & $32 \times 32 \times 1$ \\
Convolutional + ReLU & $32 \times 32 \times 1$ & 6 filters $(5 \times 5)$ & $28 \times 28 \times 6$ \\
Max Pooling & $28 \times 28 \times 6$ & $2 \times 2$ & $14 \times 14 \times 6$ \\
Convolutional + ReLU & $14 \times 14 \times 6$ & 16 filters $(5 \times 5)$ & $10 \times 10 \times 16$ \\
Max Pooling & $10 \times 10 \times 16$ & $2 \times 2$ & $5 \times 5 \times 16$ \\
Flatten & $5 \times 5 \times 16$ & - & 400 \\
Fully Connected + ReLU & 400 & 120 units & 120 \\
Fully Connected + ReLU & 120 & 84 units & 84 \\
Fully Connected & 84 & 10 units & 10 \\
Softmax & 10 & 10 units & 10 \\
\hline
\end{tabular}

LeNet-5. Thus, performing experiments based on LeNet-5 serves as a useful baseline.

The architecture of LeNet-5 used in this work is shown in Table 4. The network takes the $32 \times 32$ size images as inputs so the original MNIST images are converted to the $32 \times 32$ size in the input layer. Then, the inputs go through a convolutional layer with 6 filters followed by a max pooling layer, another convolutional layer with 16 filters followed by a max pooling layer, three fully connected layers with 120, 84 and 10 rectified linear units (ReLUs), and a softmax layer with 10 units. Note that this architecture is a variation to the original LeNet-5[10] by using ReLUs in the convolution layers and using max pooling in the sub-sampling layers.

This baseline LeNet-5 is trained on the batch size of 128 samples with a learning rate of $\eta=0.001$ for 6 epochs using the entire MNIST training set which is our clean_train. This trained clean Model A (Figure 1a) achieves a $99.05 \%$ accuracy on the entire MNIST test set which is our clean_test.

4.1.3 Cleverhans. Cleverhans is an open source library developed by Papernot et al. [13]. It provides the standard implementations of adversarial example generation methods and adversarial training. Besides, using Cleverhans can help researchers benchmark the vulnerability and accuracy of their machine learning models. There are multiple attack methods or algorithms implemented in Cleverhans. We choose four of them in this work: FGSM, JSMA, BIM, and L-BFGS. The Cleverhans version used in this work is v2.1.0.

4.1.4 Metrics. We use Success Rate to evaluate adversarial examples. It is the percentage of adversarial examples that successfully fool a model to misclassify over the total number of adversarial examples as defined in Formula 3.

$$
\text { Success Rate }=\frac{\text { number of successful adv. examples }}{\text { total number of adv. examples }}
$$

We also report Average Rate of Perturbed Features for Successful adversarial examples (ARPFS) as defined in Formula 4. Here Rate of Perturbed Features is the percentage of features changed over the total number of features in generating an adversarial example.
ARPFS $=$

$\frac{\sum \text { Rate of Perturbed Features for each successful adv. example }}{\text { total number of successful adv. examples }}$

\subsection{Adversarial Example Generation and the Existence of RGOAT}

We now provide more details about the four adversarial example generation methods. We also present the basic generation results and show that RGOAT indeed exists.

4.2.1 Fast Gradient Sign Method (FGSM). This method is proposed by Goodfellow et al. [6]. It aims to generate adversarial examples fast without constraining too much about the number and size of perturbations on an input image. Formula 5 [6] illustrates how $x_{a d v}$ is calculated:

$$
x_{a d v}=x+\epsilon \cdot \operatorname{sign}\left(\nabla_{x} J(\theta, x, y)\right)
$$

where $\theta$ represents the parameters of a trained network, and $J(\theta, x, y)$ is the cost function used to train the network. The idea behind Formula 5 is to linearize the cost function, and use the $l_{\infty}$ norm to constrain the maximum change of features.

4.2.2 Jacobian-based Saliency Map Approach (JSMA). JSMA is proposed by Papernot et al. [16] to reduce the number and size of perturbations at the cost of more intensive computation. First, the forward derivative of the network for $x_{a d v}$ (initially, $x_{a d v}$ equals to $x$ ) is computed in Formula 6 [16] to construct Adversarial Saliency Maps.

$$
S(x, t)[i]=\left\{\begin{array}{l}
0 \text { if } \frac{\partial F_{t}}{\partial x_{i}}(x)<0 \text { or } \sum_{j \neq t} \frac{\partial F_{t}}{\partial x_{i}}(x)>0 \\
\frac{\partial F_{t}}{\partial x_{i}}(x)\left|\sum_{j \neq t} \frac{\partial F_{t}}{\partial x_{i}}(x)\right| \text { otherwise }
\end{array}\right.
$$

These saliency maps indicate which input features have the most efficient influence on reaching the target class. Then, only those features are changed in the current iteration. These steps are repeated until either the target class or the maximum distortion threshold is reached.

4.2.3 Basic Iterative Method (BIM). This BIM method [8] applies FGSM $k$ times with a small step size $\alpha \geqslant \epsilon / k$, and clips the feature values of each step to ensure they are not beyond $\epsilon$ of the source example. BIM is represented in Formula 7 and Formula 8.

$$
\begin{gathered}
x_{a d v}^{0}=x \\
x_{a d v}^{N+1}=\operatorname{Clip}_{x, \epsilon}\left\{x_{a d v}^{N}+\epsilon \cdot \operatorname{sign}\left(\nabla_{x} J\left(\theta, x_{a d v}^{N}, y\right)\right)\right\}
\end{gathered}
$$

4.2.4 L-BFGS. Szegedy et al. [19] proposed the L-BFGS method to find the solution of a gradient-based optimization problem as shown in Formula 9.

$$
\begin{aligned}
& \operatorname{minimize} c \cdot\left\|x-x_{a d v}\right\|_{2}^{2}+\operatorname{loss}_{F, l}\left(x_{a d v}\right) \\
& \text { such that } x_{a d v} \in[0,1]^{n}
\end{aligned}
$$

To find the optimal value of constant $c$ which leads to the minimum $L_{2}$ distance between $x$ and $x_{a d v}$, linear search is performed. The search step and the maximum number of iterations are used to control the search progresses. 
4.2.5 Adversarial Example Generation Results and the Existence of RGOAT. We randomly selected 10 source examples for each digit from the MNIST test set. In total, the source example set contains 100 images. For each source example image, 9 adversarial examples are generated targeting at each of the 9 other digits. The same source example set is used for the four adversarial example generation methods. So for each method, 900 adversarial examples are generated. In total, the adversarial example set includes 3,600 images. Figure 2 shows examples of adversarial images generated using BIM with perturbation $\epsilon=0.3$. The rows represent source classes while the columns represent target classes. The source samples are in the diagonal.

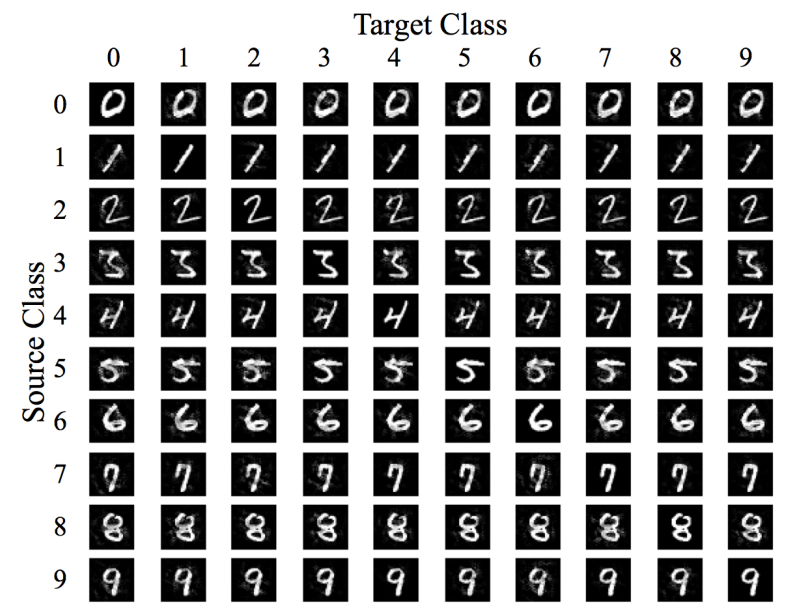

Figure 2: Adversarial examples generated using BIM with perturbation $\epsilon=0.3$.

In this work, we use the same method parameters in different models to generate adversarial examples. The parameters for the four methods are listed in Table 5 . We choose 0.3 for the perturbation $\epsilon$ in FGSM and BIM. As for JSMA, the feature change step $\theta$ is 1 , and the maximum distortion $\gamma$ is 0.1 . The maximum number of iterations for L-BFGS is 1000 . We basically choose the default parameters in Cleverhans or representative values in the research papers $[6,8,16,19]$.

Table 5: Method Parameters.

\begin{tabular}{cc}
\hline Method & Parameters \\
\hline FSGM & $\epsilon=0.3$ \\
JSMA & $\theta=1, \gamma=0.1$ \\
BIM & $\epsilon=0.3$ \\
L-BFGS & max_iterations $=1000$ \\
\hline
\end{tabular}

As shown in Figure 1a, we first train a clean Model A using LeNet-5 and the clean training set clean_train. The accuracy on the MNIST test set clean_test is $99.05 \%$. Then, we generate two sets of adversarial examples adv_A_S1 and adv_A_S2 using the same
Table 6: Success Rate and ARPFS of the adversarial examples in sets adv_A_S1 and adv_A_S2 for the Clean Model A.

\begin{tabular}{cccc}
\hline Dataset & Method & Success Rate & ARPFS \\
\hline & FSGM & 0.5544 & 0.2744 \\
adv_A_S1 & JSMA & 0.9856 & 0.1410 \\
& BIM & 0.9978 & 0.6596 \\
& L-BFGS & 1.0000 & 0.6110 \\
\hline & FSGM & 0.4956 & 0.2468 \\
adv_A_S2 & JSMA & 0.9922 & 0.1421 \\
& BIM & 0.9900 & 0.6417 \\
& L-BFGS & 1.0000 & 0.6106 \\
\hline
\end{tabular}

source examples and same model. The first set adv_A_S1 is used for adversarial training later, while the second set adv_A_S2 is used for testing the adversarially trained model. Table 6 reports the Success Rate and ARPFS of the adversarial examples in sets adv_A_S1 and adv_A_S2 for the Clean Model A. As we can see from the table, JSMA, BIM, and L-BFGS have a very high success rate. However, FGSM is a weaker attack in terms of its lower success rate because its goal is to generate adversarial examples fast but not optimally. The ARPFS for BIM is the highest while that for JSMA is the lowest.

Datasets adv_A_S1 and clean_train are used for MBEAT to train Model B as presented in Figure 1b. The accuracies of those 15 Models B listed in Table 1 on the clean test set clean_test are between $98 \%$ and $99 \%$, which are slightly lower than the accuracy of the clean Model A which is $99.05 \%$. Figure 3a illustrates the Success Rate of the adversarial examples in sets adv_B_S1 and adv_B_S2 for each Model B. The Success Rate of the adversarial examples in both sets is in general high for each Model B trained with MBEAT. Therefore, a first round adversarially trained model is not robust against the adversarial examples further generated from itself. Figure $3 b$ illustrates the ARPFS of the adversarial examples in sets adv_B_S1 and adv_B_S2, showing a similar trend as in Table 6: BIM is the highest while JSMA is the lowest. Although the ARPFS is the highest for BIM, the adversarial examples can still be perceived by human eyes as original classes as shown in Figure 2.

Model $\mathrm{C}$ shown in Figure 1c is from the second adversarial training by using datasets adv_A_S1, adv_B_S1, and clean_train. The accuracies of the four models in Table 2 on the clean test set clean_test are also between $98 \%$ and $99 \%$, which are slightly lower than the accuracy of the clean Model A which is $99.05 \%$. Table 7 shows the Success Rate and ARPFS of the adversarial examples in sets adv_C_S1 and adv_C_S2 for each Model C. The Success Rate for FGSM decreased slightly compared to 0.5544 as in Table 6. The Success Rate for the other three methods remains very high. Therefore, a second round adversarially trained model is not robust against the adversarial examples further generated from itself. Besides, ARPFS of JSMA is still very low while that of BIM is the highest. However, ARPFS of FGSM becomes lower as adversarial training is applied more times.

Model D shown in Figure 1d is from the third adversarial training by using datasets adv_A_S1, adv_B_S1, adv_C_S1, and clean_train. The accuracies of the four models in Table 3 on the clean test set clean_test are also between $98 \%$ and $99 \%$, which are slightly lower 


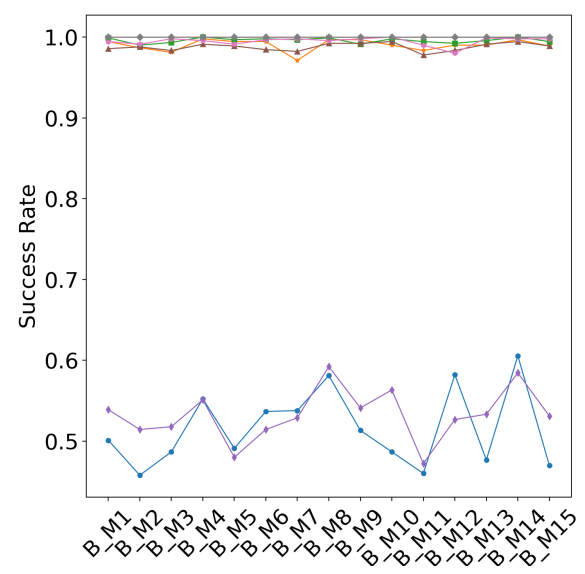

(a) Success Rate

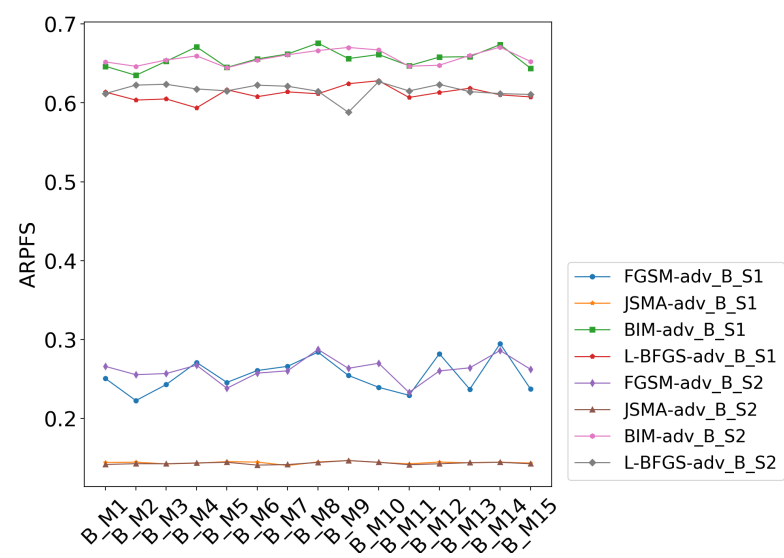

(b) ARPFS

Figure 3: Success Rate and ARPFS of the adversarial examples in sets adv_B_S1 and adv_B_S2 for each Model B.

Table 7: Success Rate and ARPFS of the adversarial examples in sets adv_C_S1 and adv_C_S2 for each Model C.

\begin{tabular}{ccccc}
\hline Dataset & Model & Method & Success Rate & ARPFS \\
\hline \multirow{4}{*}{ adv_C_S1 } & C_M1 & FSGM & 0.3144 & 0.1588 \\
& C_M2 & JSMA & 0.9822 & 0.1450 \\
& C_M3 & BIM & 0.9933 & 0.6438 \\
& C_M4 & L-BFGS & 1.0000 & 0.5970 \\
\hline \multirow{5}{*}{ adv_C_S2 } & C_M1 & FSGM & 0.2844 & 0.1434 \\
& C_M2 & JSMA & 0.9889 & 0.1441 \\
& C_M3 & BIM & 0.9900 & 0.6453 \\
& C_M4 & L-BFGS & 1.0000 & 0.6020 \\
\hline
\end{tabular}

than the accuracy of the clean Model A which is $99.05 \%$. Table 8 shows the Success Rate and ARPFS of the adversarial examples in the set adv_D_S1 for each Model D. The Success Rate for FGSM,
Table 8: Success Rate and ARPFS of the adversarial examples in the set adv_D_S1 for each Model D.

\begin{tabular}{cccc}
\hline Model & Method & Success Rate & ARPFS \\
\hline D_M1 & FSGM & 0.2956 & 0.1448 \\
D_M2 & JSMA & 0.9689 & 0.1444 \\
D_M3 & BIM & 0.9689 & 0.6190 \\
D_M4 & L-BFGS & 1.0000 & 0.5920 \\
\hline
\end{tabular}

JSMA, and BIM only decreased slightly compared to that in Table 7. Therefore, a third round adversarially trained model is not robust against the adversarial examples further generated from itself.

Overall, Models B, Models C, and Models D are the first, second, and third round adversarially trained models, respectively. We already observed in Figure 3, Table 7, and Table 8 consistently that an adversarially trained model is not robust against the adversarial examples further generated from itself (with or without MBEAT), indicating that RGOAT indeed exists.

\subsection{Method-Based Ensemble Adversarial Training}

Now we present the test results for Method-Based Ensemble Adversarial Training (MBEAT) in Figure 1b. Recall that we use the clean training set clean_train and the adversarial set adv_A_S1 to train 15 different MBEAT Models B as shown in Table 1. Figure 4 illustrates the test results of the 15 MBEAT models from B_M1 to B_M15 on the test adversarial example set adv_A_S2.

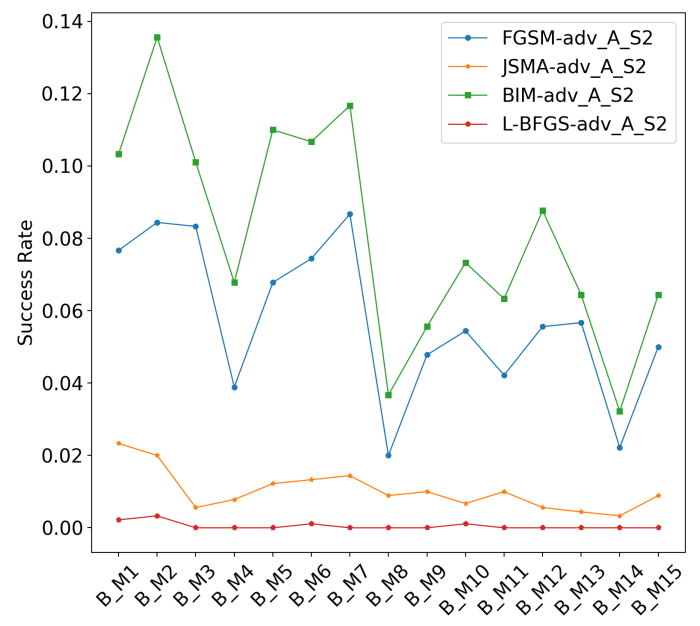

Figure 4: Success Rate of the adversarial examples in set adv_A_S2 for each Model B.

In general, all the success rates of the adversarial examples in set adv_A_S2 for Models B decreased significantly compared to the success rates of those examples for the clean Model A shown in Table 6 . Therefore, the first round adversarial training is effective against the adversarial examples generated from the clean model as expected. 
Next, let us look at the red line for L-BFGS in Figure 4. All the 15 Models $B$ are very robust against the adversarial examples generated by L-BFGS from the clean Model A. The implication is that L-BFGS is not resistant to adversarial training. From an adversary's perspective, it may not be a good idea to use L-BFGS to generate adversarial examples from the clean model for attacking an adversarially trained model.

Then, let us look at the results for B_M1 to B_M4 on the four methods in Figure 4. Each of the four models from B_M1 to B_M4 is trained with the adversarial examples generated by one method, yet the success rates for all the four methods are low. This means that a MBEAT model trained with the adversarial examples generated by one method is even robust against the adversarial examples generated by other attack methods from the clean model.

As for B_M5 to B_M10, they are trained with the adversarial examples generated by two methods. B_M5, B_M6, and B_M7 are trained with FGSM and another method. The success rates for B_M5, B_M6, and B_M7 are higher than those for B_M8, B_M9, and B_M10. B_M8 has the lowest success rates among these six models. Therefore, from a defender's perspective, adversarial training with JSMA and BIM is a good option. On the other hand, the success rates of BIM for B_M5, B_M6, and B_M7 are even higher than those for B_M1 to B_M4. The success rate of FGSM for B_7 is also higher than those for B_M1 to B_M4. Thus, it may be good to exclude FGSM for MBEAT in terms of improving the robustness of the model.

For B_M11 to B_M14, the success rates of B_M14 are the lowest. B_M14 is trained with adversarial examples from three methods excluding FGSM. Besides, the success rates of B_M14 are lower than those of B_M8. This means that adding L-BFGS to JSMA and BIM for MBEAT makes the model more robust. B_M15 is trained with all the four methods, and its success rates are higher than those of B_M14. The implication is that adding FGSM for MBEAT training does not improve the model's robustness.

In summary, we found that MBEAT is indeed beneficial in many cases, and we recommend MBEAT using adversarial examples generated by JSMA, BIM, and L-BFGS.

\subsection{Second and Third Adversarial Training}

Now, we present the test results of the second adversarial training in Figure 1c. Recall that we use the clean training set clean_train and adversarial sets adv_A_S1 and adv_B_S1 to train 4 different Models $\mathrm{C}$ in Table 2. Figure 5 illustrates the success rates of the adversarial examples in sets adv_A_S2 and adv_B_S2 for each Model C.

In Section 4.2.5, we showed that an adversarially trained model is not robust against the adversarial examples further generated from itself. Here, Figure 5 indicates that a second round adversarially trained model is still robust against the adversarial examples generated from a previous round adversarially trained Model B and the clean Model A.

Next, we present the test results of the third adversarial training in Figure 1d. Recall that we use the clean training set clean_train and adversarial sets adv_A_S1, adv_B_S1, and adv_C_S1 to train 4 different Models D in Table 3. Figure 6 illustrates the success rates of the adversarial examples in sets adv_A_S2, adv_B_S2, and adv_C_S2 for each Model D. Figure 6 further indicates that a third round adversarially trained model is still robust against the adversarial examples generated from a previous round adversarially trained Model B, Model C, and the clean Model A.

Overall, the results in Figure 5 and Figure 6 are as expected similar to those in Figure 4. This is exactly the original motivation for using adversarial training. However, what we discovered in Section 4.2.5 that RGOAT indeed exists really highlights an important limitation of adversarial training and suggests that adversarial training should be an iterative process not limited to a small number of rounds.

\section{DISCUSSIONS}

We have investigated the two research questions by performing the experiments and analyzing the experimental results. In this section, we discuss the limitations of this work and future work.

We only used four representative adversarial example generation methods for MBEAT, and did not explore other existing adversarial example generation methods. Besides, the method parameters we chose are either the default or representative ones.

In the future, more adversarial example generation methods could be used to investigate the two research questions about MBEAT and RGOAT. It could be interesting to explore more methods and also identify if there is any correlation between method parameters and the effectiveness of adversarial training.

From the defense perspective, we recommend a strong protection of the network architecture and its parameters to undermine the white-box attack capability of an adversary, recommend the use of MBEAT because it is indeed beneficial as shown in our work, and recommend the adversarial training to be iteratively performed because of the existence of RGOAT.

\section{CONCLUSION}

In this paper, we defined and explored Method-Based Ensemble Adversarial Training (MBEAT) and Round Gap Of Adversarial Training (RGOAT). We raised two research questions about MBEAT and RGOAT, and we designed an adversarial training experimental framework to answer the two questions. Our experimental results show that MBEAT is indeed beneficial, indicating that it has some important value in practice. Our experimental results also show that RGOAT indeed exists, indicating that adversarial training should be an iterative process. We made some recommendations and suggested some potential future work for further improving the effectiveness of adversarial training.

\section{ACKNOWLEDGMENTS}

Research was sponsored by the Army Research Office and was accomplished under Grant Number W911NF-17-1-0447. The views and conclusions contained in this document are those of the authors and should not be interpreted as representing the official policies, either expressed or implied, of the Army Research Office or the U.S. Government. The U.S. Government is authorized to reproduce and distribute reprints for Government purposes notwithstanding any copyright notation herein. 

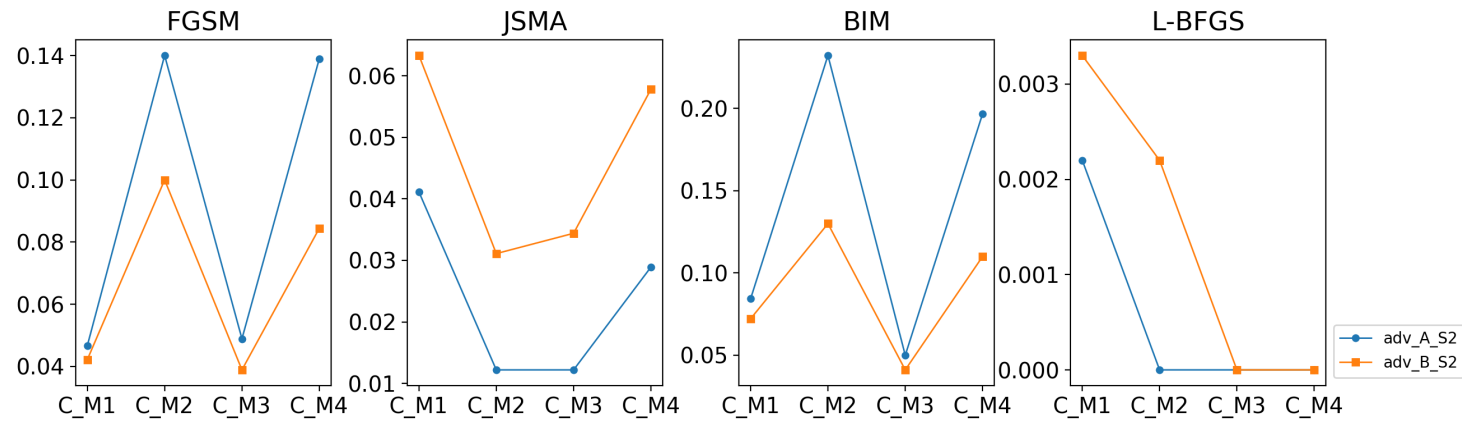

Figure 5: Success Rate of the adversarial examples in sets adv_A_S2 and adv_B_S2 for each Model C.
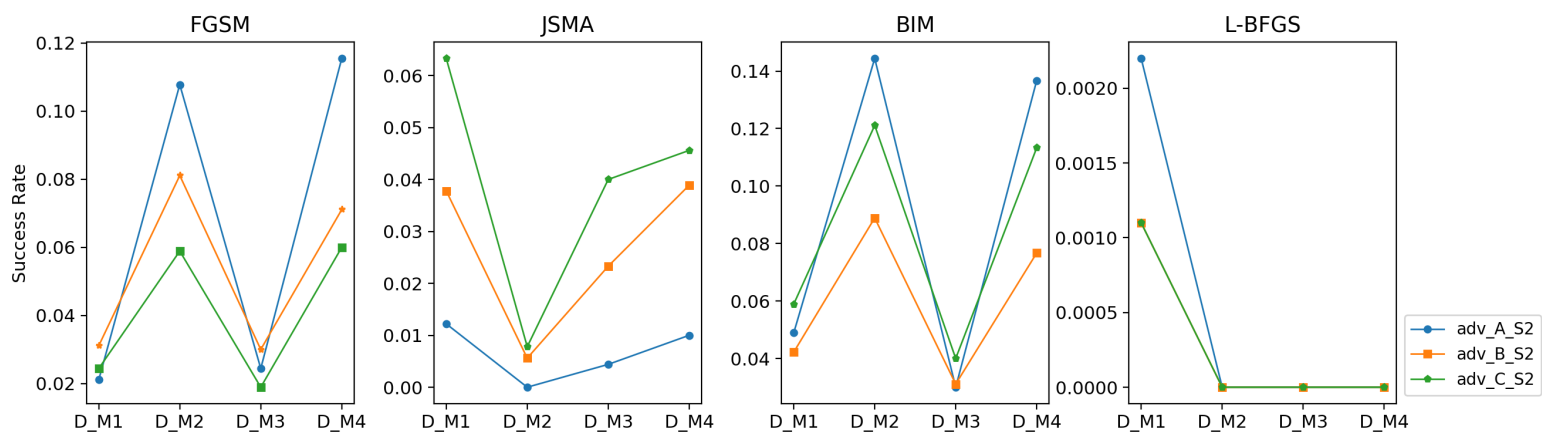

Figure 6: Success Rate of the adversarial examples in sets adv_A_S2, adv_B_S2, and adv_C_S2 for each Model D.

\section{REFERENCES}

[1] Ludwig Schmidt Dimitris Tsipras Adrian Vladu Aleksander Madry, Aleksandar Makelov. 2018. Towards Deep Learning Models Resistant to Adversarial Attacks. In International Conference on Learning Representations (ICLR).

[2] Battista Biggio, Igino Corona, Davide Maiorca, Blaine Nelson, Nedim Srndic, Pavel Laskov, Giorgio Giacinto, and Fabio Roli. 2013. Evasion Attacks against Machine Learning at Test Time. In foint European Conference on Machine Learning and Knowledge Discovery in Databases. 387-402.

[3] Nicholas Carlini and David A. Wagner. 2017. Towards Evaluating the Robustness of Neural Networks. In IEEE Symposium on Security and Privacy (SP). 39-57.

[4] Pin-Yu Chen, Yash Sharma, Huan Zhang, Jinfeng Yi, and Cho-Jui Hsieh. 2018 EAD: Elastic-Net Attacks to Deep Neural Networks via Adversarial Examples. In AAAI Conference on Artificial Intelligence (AAAI).

[5] Yinpeng Dong, Fangzhou Liao, Tianyu Pang, Hang Su, Jun Zhu, Xiaolin Hu, and Jianguo Li. 2018. Boosting Adversarial Attacks with Momentum. In IEEE Conference on Computer Vision and Pattern Recognition (CVPR).

[6] Ian J. Goodfellow, Jonathon Shlens, and Christian Szegedy. 2015. Explaining and Harnessing Adversarial Examples. In International Conference on Learning Representations (ICLR).

[7] Alex Krizhevsky, Ilya Sutskever, and Geoffrey E Hinton. 2012. ImageNet Classification with Deep Convolutional Neural Networks. In Advances in Neural Information Processing Systems 25, F. Pereira, C. J. C. Burges, L. Bottou, and K. Q. Weinberger (Eds.). Curran Associates, Inc., 1097-1105. http://papers.nips.cc/paper/ 4824-imagenet-classification-with-deep-convolutional-neural-networks.pdf

[8] Alexey Kurakin, Ian J. Goodfellow, and Samy Bengio. 2017. Adversarial Examples in the Physical World. In International Conference on Learning Representations (ICLR) Workshop Track.

[9] Alexey Kurakin, Ian J. Goodfellow, and Samy Bengio. 2017. Adversarial Machine Learning at Scale. In International Conference on Learning Representations (ICLR).

[10] Yann Lecun, Léon Bottou, Yoshua Bengio, and Patrick Haffner. 1998. Gradientbased learning applied to document recognition. In Proceedings of the IEEE. 22782324

[11] Yann LeCun and Corinna Cortes. 2010. MNIST handwritten digit database http://yann.lecun.com/exdb/mnist/. (2010). http://yann.lecun.com/exdb/mnist/

[12] Seyed-Mohsen Moosavi-Dezfooli, Alhussein Fawzi, and Pascal Frossard. 2016. DeepFool: a simple and accurate method to fool deep neural networks. (2016).
[13] Nicolas Papernot, Fartash Faghri, Nicholas Carlini, Ian J. Goodfellow, Reuben Feinman, Alexey Kurakin, Cihang Xie, Yash Sharma, Tom Brown, Aurko Roy, Alexander Matyasko, Vahid Behzadan, Karen Hambardzumyan, Zhishuai Zhang, Yi-Lin Juang, Zhi Li, Ryan Sheatsley, Abhibhav Garg, Jonathan Uesato, Willi Gierke, Yinpeng Dong, David Berthelot, Paul Hendricks, Jonas Rauber, Rujun Long, and Patrick McDaniel. 2018. Technical Report on the CleverHans v2.1.0 Adversarial Examples Library. CoRR abs/1610.00768 (2018). arXiv:1610.00768 http://arxiv.org/abs/1610.00768

[14] Nicolas Papernot, Patrick McDaniel, Ian Goodfellow, Somesh Jha, Z. Berkay Celik, and Ananthram Swami. 2017. Practical Black-Box Attacks Against Machine Learning. In Asia Conference on Computer and Communications Security. 506-519.

[15] Nicolas Papernot, Patrick D. McDaniel, and Ian J. Goodfellow. 2016. Transferability in Machine Learning: from Phenomena to Black-Box Attacks using Adversarial Samples. CoRR abs/1605.07277 (2016). arXiv:1605.07277 http: //arxiv.org/abs/1605.07277

[16] Nicolas Papernot, Patrick D. McDaniel, Somesh Jha, Matt Fredrikson, Z. Berkay Celik, and Ananthram Swami. 2016. The Limitations of Deep Learning in Adversarial Settings. In IEEE European Symposium on Security and Privacy. 372-387.

[17] Sara Sabour, Yanshuai Cao, Fartash Faghri, and David J. Fleet. 2016. Adversarial Manipulation of Deep Representations. In International Conference on Learning Representations (ICLR).

[18] Christian Szegedy, Wei Liu, Yangqing Jia, Pierre Sermanet, Scott Reed, Dragomir Anguelov, Dumitru Erhan, Vincent Vanhoucke, and Andrew Rabinovich. 2015. Going Deeper with Convolutions. In Computer Vision and Pattern Recognition (CVPR). http://arxiv.org/abs/1409.4842

[19] Christian Szegedy, Wojciech Zaremba, Ilya Sutskever, Joan Bruna, Dumitru Erhan, Ian J. Goodfellow, and Rob Fergus. 2013. Intriguing Properties of Neural Networks. CoRR abs/1312.6199 (2013). arXiv:1312.6199 http://arxiv.org/abs/1312.6199

[20] Florian Tramèr, Alexey Kurakin, Nicolas Papernot, Ian J. Goodfellow, Dan Boneh, and Patrick McDaniel. 2018. Ensemble Adversarial Training: Attacks and Defenses. In International Conference on Learning Representations (ICLR).

[21] Jonathan Uesato, Brendan O'Donoghue, Aäron van den Oord, and Pushmeet Kohli. 2018. Adversarial Risk and the Dangers of Evaluating Against Weak Attacks. In International Conference on Machine Learning (ICML).

[22] Weilin Xu, Yanjun Qi, and David Evans. 2016. Automatically Evading Classifiers: A Case Study on PDF Malware Classifiers. In Network and Distributed System Security Symposium (NDSS). 